\section{The first sugar of the repeat units is essential for the Wzy polymerase activity and elongation of the $\mathrm{O}$-antigen lipopolysaccharide}

\author{
Susana Merino', Victor Gonzalez² \& Juan M Tomás*,1
}

\begin{abstract}
Aim: In the Wzx/Wzy-dependent assembled pathway, the assembled O-antigen repeat units are translocated by a Wzx translocase and then, polymerized by the integral membrane protein Wzy to form a glycan chain. In this study, we investigate the relation between the first sugar of the O-antigen repeat unit and the Wzy polymerase. Material \& methods: Crosscomplementation studies in two different reducing/non-reducing end affinity systems were performed. Results \& conclusion: We demonstrate that the activity of the Escherichia coli $\mathrm{O}$-antigen polymerase (Wzy) is dependent on the first sugar of the $\mathrm{O}$-antigen repeat unit to produce the $\mathrm{O}$-antigen polymerization and therefore, there is a need for a concerted action with the enzyme transferring the initial HexNAc to undecaprenol phosphate. Furthermore, polymerization activity of Aeromonas hydrophila Wzy-O34 is permissive with the sugar at the non-reducing end of the $\mathrm{O}$-antigen repeat unit.
\end{abstract}

First draft submitted: 18 December 2015; Accepted for publication: 6 May 2016;

Published online: 30 June 2016

Bacterial cell surface polysaccharides play important roles in processes critical for bacterial pathogenicity and environmental adaptations. Lipopolysaccharide (LPS) is the major glycoconjugate constituent of the outer leaflet of the outer membrane (OM), in Gram-negative bacteria. LPS molecules comprise three chemically distinct regions: lipid A, a hydrophobic glycolipid which anchors LPS in the bacterial membrane; core oligosaccharide (OS), a nonrepeating oligosaccharide which usually contains heptoses and keto-deoxyoctulosonic acid; and the $\mathrm{O}$-specific polysaccharide (O-antigen), a glycan chain of homo- or hetero-polysaccharide repeat units [1]. In general, variations of lipid A and core oligosaccharide are relatively limited. However, the chemical composition and structure of $\mathrm{O}$-antigens vary widely among Gram-negative bacteria, leading to a large number of $\mathrm{O}$-serotypes [2] . For example, there are over 186 O-antigen forms for Escherichia coli [3].

Four major modes of O-antigen assembly have been defined: the Wzm/Wzt-, the Wzx/Wzy-, the synthetase- and the Wzk-dependent pathways [4]. Nearly all E. coli $\mathrm{O}$-antigens are synthesized by the Wzx/Wzy-dependent pathway [5], as is in Aeromonas hydrophila O34 [6]. This pathway starts at the cytosolic face of the inner membrane by the formation of a linkage between the lipid carrier undecaprenyl phosphate (Und-P) and the first sugar 1-phosphate, of the corresponding $\mathrm{O}$-antigen unit, transferred from a sugar nucleoside diphosphate. Two different classes of integral membrane protein are able to transfer the first sugar onto Und-P to give Und-PP-linked sugar: the $N$-acetylhexosamine-1phosphate transferase and the polyisoprenyl-phosphate hexose-1-phosphate transferase. In E. coli, the initial transferase is usually WecA, which transfers $N$-acetylglucosamine-phosphate (GlcNAc-1-P)

\section{KEYWORDS}

- Escherichia coli • O-antigen polymerization $\bullet$ Wzy

O-antigen assembly

pathway

'Departamento de Microbiología, Facultad de Biología, Universidad de Barcelona, Diagonal 643, 08071 Barcelona, Spain

${ }^{2}$ Institute of Virology, Hannover Medical School, Hannover, Germany

*Author for correspondence: Tel.: +34 934021486; Fax: +34 934039047; jtomas@ub.edu

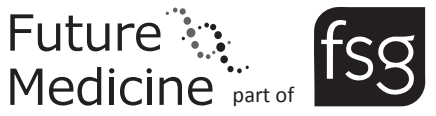


to Und-P to give Und-PP-linked GlcNAc [5]. In $A$. hydrophila AH-3, the initial transferase is WecP, which transfers $N$-acetylgalactosaminephosphate (GalNAc-1-P) to Und-P to give UndPP-linked GalNAc [7]. The remaining sugars of the $\mathrm{O}$-antigen repeat unit are subsequently transferred from nucleotide-sugar precursors to the Und-PP-linked sugar by a serial action of glycosyltransferases. Completed $\mathrm{O}$ units are flipped across the inner membrane by the integral membrane protein Wzx [8,9] and then assembled on the periplasmic side of the inner membrane by the Wzy O-antigen polymerase [10]. Wzx and Wzy both exhibit enormous sequence diversity. The chain length of the final polymer is regulated by the $\mathrm{O}$-antigen chain length regulator Wzz [11]. Finally, the O-antigen polymer is ligated to the lipid A-core OS by an $\mathrm{O}$-antigen ligase (WaaL) [12] and complete LPS molecule is translocated from the inner membrane to the outer leaflet of the outer membrane by the Lpt proteins [13].
The O-antigen polymerization reaction generates a glycosidic linkage between the nonreducing end of a new $\mathrm{O}$-antigen unit linked to Und-PP, to the reducing end of the Und-PP-linked growing polymer [14,15]. Wzy O-antigen polymerases are very hydrophobic integral membrane proteins that contain 10-14 transmembrane domains with two major periplasmic loops. Even at the amino acid level, they show little similarity to each other in terms of primary sequence, making the identification of catalytic and binding residues difficult [14]. The polymerase genes are located in the gene clusters involved in the polysaccharide biosynthesis that polymerize.

The O-antigen units of E. coli O7, O150 and O159, as well as $A$. hydrophila $\mathrm{O} 34$, are synthesized individually at the cytosolic side of the inner membrane by the Wzx/Wzy-dependent assembly pathway. Each repeat unit is translocated across the cytoplasmic membrane by the Wzx (O-unit flippase) and then, it needs to be polymerized. The O-antigen polymerase (Wzy)

\section{Table 1. Bacterial strains and plasmids used.}

Strains or plasmids Relevant characteristics

Source or ref.

\begin{tabular}{|c|c|c|}
\hline \multicolumn{3}{|l|}{ Escherichia coli strains } \\
\hline $\mathrm{DH} 5 \alpha$ & $\begin{array}{l}\mathrm{F}^{-} \text {end } \mathrm{A} \text { hsdR17 }\left(\mathrm{rK}^{-} \mathrm{mK} \mathrm{K}^{+}\right) \text {supE44 thi-1 recA1 gyr- } \\
\text { A96_80lacZM15 }\end{array}$ & {$[17]$} \\
\hline XL1-Blue & $\begin{array}{l}\text { recA1 endA1 gyrA96 thi-1 hsdR17supE44 relA1 lac (F-proAB } \\
\text { laclqZ_M15 Tn10) }\end{array}$ & Stratagene \\
\hline VW187 & O7:K1; clinical isolate & {$[18]$} \\
\hline MV501 & VW187; wecA::Tn10 Tc ${ }^{R}$ & {$[18]$} \\
\hline 0150 & O25b-ST131 (serotype O150) & {$[19]$} \\
\hline 0159 & CCUG36899 (serotype 0159) & [19] \\
\hline 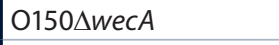 & O25b-ST131 wecA mutant in frame with pKO3 & This study \\
\hline 0159 & CCUG36899 wecA mutant in frame with pKO3 & This study \\
\hline \multicolumn{3}{|l|}{ Aeromonas strains } \\
\hline $\mathrm{AH}-405$ & AH-3, spontaneous Rif ${ }^{R}$ & {$[20]$} \\
\hline $\mathrm{AH}-405 \Delta w z y$ & $\mathrm{AH}-405$ wzy mutant in frame with pDM4 & {$[6]$} \\
\hline \multicolumn{3}{|l|}{ Plasmids } \\
\hline pRK2073 & Helper plasmid, $S p c^{R}$ & {$[6]$} \\
\hline pKO3 & $\mathrm{Cm}^{\mathrm{R}} \mathrm{s} a c B$ temperature sensitive suicide vector & {$[21]$} \\
\hline $\mathrm{pKO} 3 \Delta w e c A_{0150}$ & pKO3 with wecA in frame deletion & This study \\
\hline pGEMT-Gne ${ }_{A h}$ & pGEM-T vector with complete gne of $\mathrm{AH}-3$ & {$[22]$} \\
\hline pBAD33 & Arabinose inducible expression vector, $\mathrm{Cm}^{\mathrm{R}}$ & ATCC \\
\hline pBAD33-Gm & pBAD33 vector with $\mathrm{Gm}^{\mathrm{R}}$ & {$[23]$} \\
\hline pBAD33-WecP ${ }_{\text {Ah }}$ & pBAD33-Gm with Aeromonas hydrophila AH-3 wecP & {$[7]$} \\
\hline pBAD33-WecA $A_{E c}$ & pBAD33-Gm with Escherichia coli VW187 wecA & {$[7]$} \\
\hline pBAD-WecP-Wzy ${ }_{\text {Ah }}$ & pBAD33-WecP ${ }_{\text {Ah }}$ with A. hydrophila AH-3 wzy & {$[24]$} \\
\hline pBAD-WecA-WzyE $E_{\mathrm{Ec}}$ & $\begin{array}{l}\text { pBAD } 33-\text { WecA } A_{E c} \text { with } E \text {. coli DH5 } \alpha \text { wzy enterobacterial } \\
\text { common antigen }\end{array}$ & {$[24]$} \\
\hline$p B A D-W z y E_{E c}$ & $\begin{array}{l}\text { pBAD33 with } E \text {. coli DH5 } \alpha \text { wzy enterobacterial common } \\
\text { antigen }\end{array}$ & {$[24]$} \\
\hline
\end{tabular}


transfers the growing chain to the nonreducing end of the new O-subunit, establishing a glycosidic bond. The concerted action of Wzy and Wzz (O-antigen chain length regulator) is responsible for the polymerization of the $\mathrm{O}$-antigen units to a certain length distribution that is unique to each O-antigen [16]. In the current study, we show that E. coli O-antigen polymerase has specificity for the first sugar of the $\mathrm{O}$-antigen repeat unit and, therefore, the concerted action of the enzyme mediating the transfer of HexNAc to Und-P and the $\mathrm{O}$-antigen polymerase (Wzy) is involved in the $\mathrm{O}$-antigen polymerization by the $\mathrm{Wzx} / \mathrm{Wzy}$ dependent $\mathrm{O}$-antigen export and assembly pathway. Furthermore, $A$. hydrophila Wzy-O34 polymerization activity is permissive with the sugar at the nonreducing end of the $\mathrm{O}$-antigen repeat unit.

\section{Materials \& methods}

\section{- Bacterial strains, plasmids \& growth}

\section{conditions}

Bacterial strains and plasmids used are shown in Table 1. Escherichia coli were grown either in Miller lysogeny broth (LB) and LB Miller agar. Aeromonas were grown either in tryptic soy broth (TSB) or tryptic soy agar (TSA). Chloramphenicol $(25 \mu \mathrm{g} / \mathrm{ml})$, gentamicin $(20 \mu \mathrm{g} / \mathrm{ml})$ or ampicillin $(100 \mu \mathrm{g} / \mathrm{ml})$ were added to the different media when required.

\section{- General DNA methods}

Standard DNA manipulations were done as described [25]. DNA restriction endonucleases, T4 DNA ligase, E. coli DNA polymerase (Klenow fragment) and alkaline phosphatase were used as recommended by the suppliers.

\section{- DNA sequencing \& computer analysis of sequence data}

Double-stranded DNA sequencing was performed by using the dideoxy-chain termination method [26] with the BigDye Terminator v3.1 cycle sequencing kit (Applied Biosystems). Oligonucleotides used for genomic DNA amplifications and DNA sequencing were designed from GenBank EU274663.1, DQ119103.1, CP003034.1, NC_007779.1 or AP009378, and purchased from Sigma-Aldrich. Deduced amino acid sequences were compared with those of DNA translated in all six frames from nonredundant GenBank and EMBL databases by using the BLAST [27] network service at the National Center for Biotechnology Information and European Biotechnology Information.
ClustalW was used for multiple-sequence alignments [28] and the topology maps were predicted by using TMMTOP 2.0 [29].

\section{- Mutant construction}

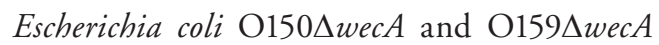
mutants were constructed by creating an in vitro in-frame deletion of the gene [21], as previously described [30]. Escherichia coli O25b-ST131 (O150) and CCUG 36899 (O159) strains were used [19]. The mutant was constructed using E. coli O150 chromosomal DNA and primers Ecrfe-A2wecA-A (5'-GGAAGATCTGTGTTGACCGGTGGTGTGG-3'), Ecrfe-BwecA-B (5'-CCCATCCACTAAACTTAAACACT GGTGACGTTTGCGGAAGT-3'), EcrfeCwecA-C (5'-TGTTTAAGTTTAG TGGATG GGGGTGGCAGCCCCAAT T TAAC $\left.-3^{\prime}\right)$, and Ecrfe-D2wecA-D (5'-GGAAGA TCTATCCGGCGGTCATAGATGG-3') in two sets of asymmetric PCRs to amplify DNA fragments $A B$ and $C D$, respectively. DNA fragments $A B$ and $C D$ were annealed at their overlapping region (underlined letters in primers $\mathrm{B}$ and $\mathrm{C}$ ) and amplified by PCR as a single fragment, using primers $A$ and $D$. The fusion product was purified, BglII-digested (double-underlined letters in primers A and D), ligated into BamHI-digested and phosphatase-treated $\mathrm{pKO} 3$ vector, electroporated into $E$. coli $\mathrm{DH} 5-\alpha$, and plated on chloramphenicol LB agar plates at $30^{\circ} \mathrm{C}$ to obtain plasmid pKO3 $\Delta w_{e c} A_{\mathrm{O} 150}$. The mutated gene was transferred to the chromosome by homologous recombination using the temperature-sensitive suicide plasmid pKO3, containing the counterselectable marker $s a c B$. The plasmid containing the engineered inframe deletion ( $\mathrm{pKO} 3 \Delta w e c A_{O 150}$ ) was transferred into $E$. coli $\mathrm{O} 150$ and $\mathrm{O} 159$ strains by transformation. Mutants were independently selected based on growth on LB agar containing 10-15\% sucrose and loss of the chloramphenicol resistance marker of vector pKO3 [21]. The mutations in each strain were confirmed by sequencing of the whole constructs in amplified PCR products.

\section{- Complementation studies}

Plasmids pBAD33-WecP ${ }_{\mathrm{Ah}}$, pBAD33-WecA $\mathrm{Ec}_{\mathrm{c}}$ or pBAD-WecP-W $\mathrm{Zy}_{\mathrm{Ah}}$ were independently transferred into the $E$. coli wecA mutants by electroporation. Plasmid $\mathrm{pBAD}-\mathrm{WzyE}_{\mathrm{Ec}}$ was transferred into the $A$. hydrophila AH-3 wzy mutant by triparental mating using the mobilizing strain HB101/ pRK20173. Transformants were selected on plates containing gentamycin or chloramphenicol and 
confirmed by PCR. Each gene was expressed from the arabinose-inducible and glucose-repressible pBAD33 promoter $\left(\mathrm{P}_{\mathrm{BAD}}\right)$. Repression from the ara $C$ promoter was achieved by growth in medium containing $0.2 \%$ (w/v) $D$-glucose and induction was obtained by adding $L$-arabinose to a final concentration of $0.2 \%(\mathrm{w} / \mathrm{v})$ [31]. Plasmid pGEMT-Gne was transferred into the E. coli wec $A$ mutants by electroporation. Transformants were selected on plates containing ampicillin and confirmed by PCR.

\section{- LPS isolation \& electrophoresis}

Cells were grown in LB or TSB, washed with water and dehydrated by sequential washing with methanol:chloroform $(1: 1) \times 3$, ethanol, acetone $\times 2$ and diethyl ether. After evaporation of the last dissolvent, the LPS was extracted from the dehydrated cells. For strains producing LPS without O-antigen chains, the phenol/chloroform/light petroleum ether method [32] was used, while the phenol/water procedure [33] was used for strains producing a complete LPS (with O-antigen domain). For screening purposes, LPS was obtained from whole cells treated with proteinase $\mathrm{K}$ [34]. LPS samples were separated by SDS-PAGE or N-(2-hydroxy-1, 1-bis [hydroxymethyl] ethyl) glycine (Tricine)-SDS-PAGE (sodium dodecyl sulfate-polyacrylamide gel electrophoresis) and visualized by silver staining [35].

\section{- Preparation of oligosaccharides}

The LPS preparations (20 mg) were hydrolyzed in $1 \%$ acetic acid $\left(100^{\circ} \mathrm{C}, 120 \mathrm{~min}\right)$ and centrifuged $(8000 \times g, 30 \mathrm{~min})$. To obtain the lipid A, the precipitates were collected and lyophilized. The oligosaccharide fractions: high-molecularmass polysaccharide (PS), LPS core-oligosaccharide (OS) and sometimes intermediate fractions were obtained by fractionation of the supernatants. Fractions of supernatants were obtained on a column $(56 \times 2.6 \mathrm{~cm})$ of Sephadex G-50 (S) in $0.05 \mathrm{M}$ pyridinium acetate buffer, $\mathrm{pH} 4.5$ and monitored by a differential refractometer.

\section{- Gas chromatography-mass spectrometry analysis}

The PS fraction was hydrolyzed with two $\mathrm{M}$ $\mathrm{CF}_{3} \mathrm{CO}_{2} \mathrm{H}$ for $2 \mathrm{~h}$ at $100^{\circ} \mathrm{C}$, and the monosaccharides were conventionally converted into methylated alditol acetates and methyl glycoside acetates. The converted sugars were analyzed on an Agilent Technologies 5973N MS instrument equipped with a 6850A GC and an RTX-5 capillary column (Restek, $30 \mathrm{~m} \times 0.25 \mathrm{~mm}$ id, flow rate $1 \mathrm{ml} / \mathrm{min}$, He as carrier gas). Analysis of acetylated methyl glycosides was performed with the following temperature program: 150 for $5 \mathrm{~min}$, $150 \rightarrow 250$ at $3^{\circ} \mathrm{C} / \mathrm{min}, 250$ for $10 \mathrm{~min}$. The program used for partially methylated alditol acetates was: $90^{\circ} \mathrm{C}$ for $1 \mathrm{~min}, 90^{\circ} \mathrm{C} \rightarrow 140^{\circ} \mathrm{C}$ at $25^{\circ} \mathrm{C} / \mathrm{min}$, $140^{\circ} \mathrm{C} \rightarrow 200^{\circ} \mathrm{C}$ at $5^{\circ} \mathrm{C} / \mathrm{min}, 200^{\circ} \mathrm{C} \rightarrow 280^{\circ} \mathrm{C}$ at $10^{\circ} \mathrm{C} / \mathrm{min}, 280^{\circ} \mathrm{C}$ for $10 \mathrm{~min}$.

\section{- Isolation \& purification of enterobacterial common antigen}

The procedure was employed as previously described [36]. Briefly, bacterial cells were extracted by a combined hot phenol-water extraction (9-48) and phenol-chloroform-petroleum (PCP) ether fractionation (8-11). After dialysis and lyophilization, the aqueous phase of the phenol-water extraction was treated with the PCP mixture. The LPS precipitated when some drops of water were added to the phenol phase, while enterobacterial common antigen (ECA) remained in solution and was subsequently recovered by extensive dialysis and lyophilization. This fraction was resuspended in water and centrifuged at $105,000 \times g$ for $4 \mathrm{~h}$. The lyophilized supernatant is enriched in ECA and was further purified by column chromatography on diethylaminoethyl-cellulose with stepwise elution with $0.5,1.0$ and $1.6 \mathrm{M}$ ammonium acetatemethanol buffer [37]. ECA is eluted in the middle fraction and, after dialysis was lyophilized. For quantitative determination of mannosaminuronic acid, the ECA fraction was reduced with $\mathrm{NaBH} 4$ after reaction with the carbodiimide reagent and hydrolysis with $4 \mathrm{M} \mathrm{HC1}$ for 6 h. Sugars $(\mathrm{GlcN}$ and $\mathrm{ManN}$ ) in the reduced polymer were found to be substrates for enzymatic phosphorylation by hexokinase/ATP [38].

\section{- Deoxycholate sensitivity assay}

Five-microliter portions of the appropriate dilutions of exponential cultures of the wild-type and mutant strains were incubated for $24 \mathrm{~h}$ at $37^{\circ} \mathrm{C}$ on an LB plate or an LB plate containing 1\% deoxycholate (DOC). The minimum inhibitory concentration (MIC) was calculated according to their inability to grow on LB plates containing several DOC concentrations. Similar amount of bacteria were inoculated in the original drops.

\section{Results}

Escherichia coli has so many O-antigen serogroups, but majority are Wzy-dependent and the initial sugar of the repeat subunit is $N$-acetyl-D-glucosamine 
(D-GlcNAc). When D-GlcNAc is not present, $N$-acetyl-D-galactosamine (D-GalNAc) takes its place. Only in two strains (O45 and O62D1), $N$-acetyl-D-fucosamine (D-FucNAc) has been found at the reducing end [5]. To study the relation between first sugar of the $\mathrm{O}$-antigen repeat unit and the Wzy polymerase, we need two different affinity systems. Given the O:34-antigen repeat unit of $A$. hydrophila AH-3 have D-GalNAc at the reducing end (Figure 1A), we decided to use E.coli O-antigen serogroups with D-GlcNAc at the reducing end of repeat unit. The sugar variability at the nonreducing end of E. coli serogroups is too high; therefore, we used three serogroups with different sugars at the nonreducing-end: $\mathrm{O} 7$ has 4-acetamido-4, 6-dideoxyD-glucose (D-Qui4NAc); and O150 and O159 have D-GlcNAc (Figure 1B \& D). Furthermore, we used the ECA, which has D-GlcNAc at the reducing end and 4-acetamido-4, 6-dideoxy-Dgalactose (D-Fuc4NAc) at the nonreducing end
(Figure 1E). In E. coli O7, O150 and O159, the transfer of D-GlcNAc to Und-P is performed by WecA [5], whose gene encoding is in the ECA cluster. In $A$. hydrophila $\mathrm{O} 34$ the proximal sugar is D-GalNAc (Figure 1A), and the transfer of this sugar to Und-P is performed by WecP [7], whose gene encoding is in the $\mathrm{O}$-antigen cluster. WecA and WecP are both UDP-HexNAc:polyprenol-P HexNAc-1-P transferases.

\section{- Cross-complementation studies in E. coli MV501 (wecA mutant from serotype 07)}

The E. coli MV501 mutant is unable to add the initial sugar D-GlcNAc to the Und-P and therefore, is unable to biosynthesize the O7-antigen subunit (Figure 2, Lane 2) [18]. When plasmid pBAD33-WecA $\mathrm{Ec}_{\mathrm{Ec}}$ (carrying the E. coli VW187 [O7] wecA) was introduced in E. coli MV501 mutant and expressed with arabinose, we could see identical LPS profile as their wild-type strain, E. coli VW187 serotype O7 (Figure 2, Lanes 5

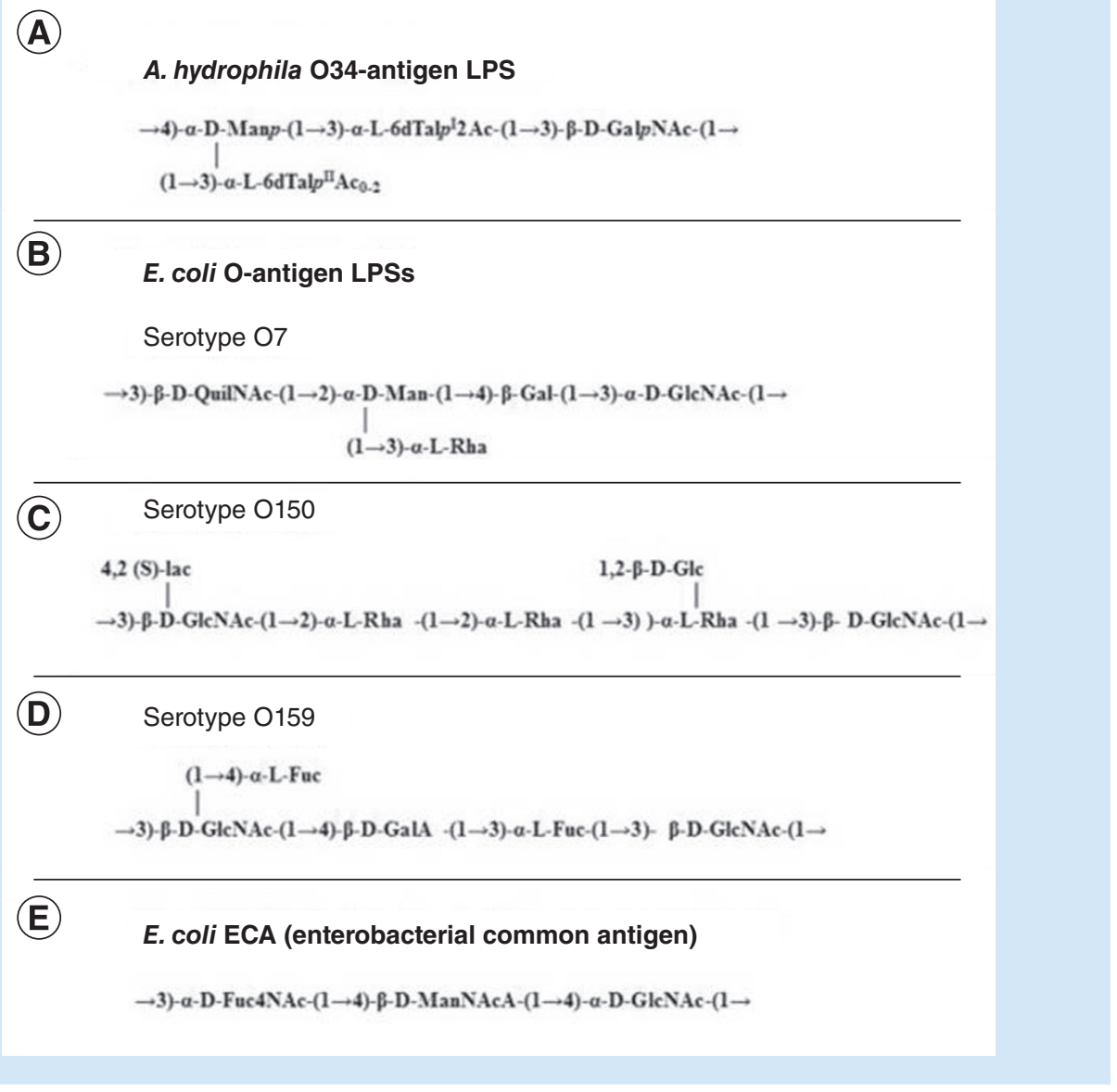

Figure 1. Chemical structures. (A) Aeromonas O34-antigen LPS [39], (B) Escherichia coli O-antigen LPS from serotypes 07 [40], (C) 0150 [41] and (D) 0159 [42] and (E) E. coli ECA [43].

ECA: Enterobacterial common antigen; LPS: Lipopolysaccharide. 
(A)

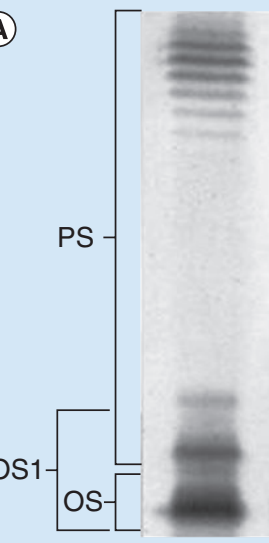

1

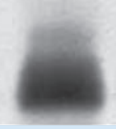

2

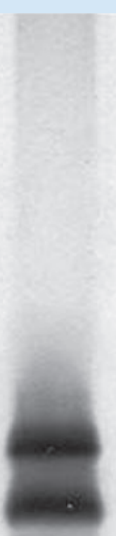

3
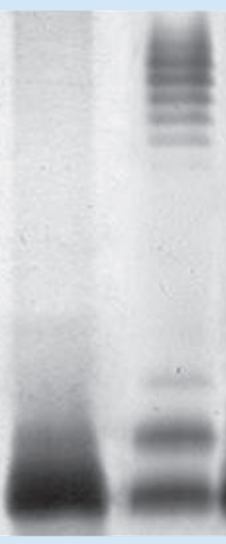

4

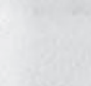

(B)

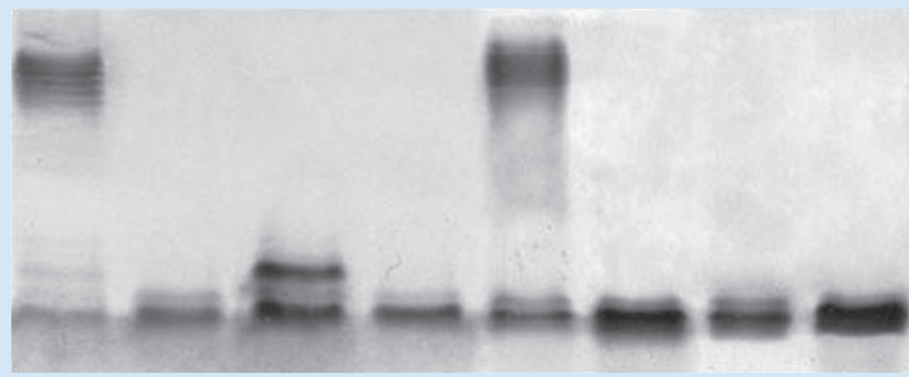

\begin{tabular}{|c|c|c|c|c|}
\hline Lane & Strain & $\begin{array}{l}\text { Gene } \\
\text { knocked }\end{array}$ & Gene introduced & Plasmid \\
\hline 1 & VW187 & & & \\
\hline 2 & VW187 & wecA & & \\
\hline 3 & VW187 & wec $A$ & ${ } e_{e c} P_{\mathrm{Ah}} / g n e \mathrm{AH}-3$ & pBAD33-Gm/pGEMTeasy \\
\hline 4 & VW187 & wecA & $w_{e c} P_{A \mathrm{~A}} w z y_{\mathrm{An}} / g n e \mathrm{AH}-3$ & pBAD33-Gm/pGEMTeasy \\
\hline 5 & VW187 & wec $A$ & $w e c A_{E c} V W 187$ & pBAD33-Gm \\
\hline 6 & VW187 & wec $A$ & $w e c P_{\mathrm{An}} \mathrm{AH}-3$ & pBAD33-Gm \\
\hline 7 & VW187 & wecA & $w e c P w z y_{A h} \mathrm{AH}-3$ & pBAD33 \\
\hline 8 & VW187 & wecA & gne $\mathrm{AH}-3$ & pGEMTeasy \\
\hline
\end{tabular}

Figure 2. Polyacrylamide gels showing the migration of lipopolysaccharide from Escherichia coli MV501 (wecA mutant) and its complementation. The lipopolysaccharide samples were separated on SDS-PAGE (A) and SDS-Tricine-PAGE (B) and visualized by silver staining. Shown are lipopolysaccharide samples from E. coli VW187 (wild-type) (Lane 1), MV501 (Lane 2), MV501 + pBAD33-WecP and pGEMTGne (Lane 3), MV501 + pBAD-WecP-Wzy ${ }_{\text {Ah }}$ and pGEMT-Gne ${ }_{\text {Ah }}$ (Lane 4), MV501 + pBAD-WecA EC $_{\text {(Lane }}$ 5), pBAD-WecP ${ }_{\text {Ah }}$ (Lane 6), pBAD-WecP-Wzy ${ }_{\text {Ah }}$ (Lane 7) and MV501 + pGEMTeasy-Gne ${ }_{A h}\left(\right.$ Lane $_{\text {8) }}$. Lipopolysaccharide fractions: OS, core oligosaccharide fraction; OS1, core oligosaccharide with a single repeat unit; and PS, $\mathrm{O}$-antigen fraction. All the strains harboring PBAD plasmids were grown under induced conditions (+ arabinose) as indicated in the 'Materials \& methods' section.

$\& 1$ ). According to their LPS profiles on gels, neither the plasmid vector alone nor plasmid pBAD$\mathrm{WecP}_{\mathrm{Ah}}$ (carrying the $A$. hydrophila $\mathrm{AH}-3$ wec $P$ ) fully complemented the mutant under expressing conditions (+ arabinose) (Figure 2, Lane 6).

In $A$. hydrophila $\mathrm{AH}-3$, WecP transfers D-GalNAc to Und-P. To obtain this initial sugar, Aeromonas require the activity of the Gne enzyme, which is a UDP-GalNAc4-epimerase responsible for the conversion of UDP-GlcNAc to UDP-GalNAc [22]. When plasmid pBAD33$\mathrm{WecP}_{\mathrm{Ah}}+$ pGEMT-Gne ${ }_{\mathrm{Ah}}$ was introduced in E. coli MV501 mutant and expressed with arabinose, it showed an LPS profile on gels with 
two bands (one with the same mobility as the mutant and the other one with a reduced mobility) (Figure 2, Lane 3). However, no changes could be observed when the mutant carries the plasmid pGEMT-Gne ${ }_{\mathrm{Ah}}$ alone (Figure 2, Lane 8). LPS isolated from E. coli MV501 mutant with plasmids pBAD33-WecP ${ }_{\mathrm{Ah}}+\mathrm{pGEMT}$ $\mathrm{Gne}_{\mathrm{Ah}}$ together grown under expressing conditions (+ arabinose) showed by sugar analysis a single $\mathrm{O}$-antigen repeat unit in the E. coli $\mathrm{O} 7$ background which contain D-GalNAc (Figure 3).

When we introduced plasmid pBAD-WecP$\mathrm{Wzy}_{\mathrm{Ah}}$ into E. coli MV501 mutant with or without plasmid pGEMT-Gne ${ }_{\mathrm{Ah}}$, and grown under expressing conditions (+ arabinose) both showed an LPS profile on gels with a single band with a mobility identical to the E. coli MV501 mutant alone (Figure 2, Lanes $4 \& 7$ ). It seems that the introduction of wzy from $A$. hydrophila $\mathrm{AH}-3$ (serotype O34) causes the loss of the additional LPS band with reduced mobility observed in the same mutant strain without this gene. The sugar analysis of LPS isolated from E. coli MV501 mutant with plasmids pBAD-WecP$\mathrm{Wzy}_{\mathrm{Ah}}+\mathrm{pGEMT} \mathrm{Gne}_{\mathrm{Ah}}$ together, grown under expressing conditions ( + arabinose), shown the lack of monosaccharide mannose in the E. coli O7-antigen LPS [44], which means the loss of the single O7-antigen LPS repetition (Figure 3). To evaluate the ability of $\mathrm{Wzy}_{\mathrm{Ah}}$ to polymerize other $E$. coli surface polysaccharides and given that $w e c A$ and $w z y E_{\mathrm{Ec}}$ belong to the ECA gene cluster [45], we analyzed the ability of complemented mutants to produce ECA. It has been described that mutants unable to produce ECA showed sensitivity to DOC [46]. We tested the MIC for DOC as it is shown in Table 2. The wild-type strain is resistant to DOC, while E. coli MV501 (wecA mutant) is sensitive. The introduction in the MV501 mutant of pBAD33$\mathrm{WecP}_{\mathrm{Ah}}+\mathrm{pGEMT}_{\mathrm{G}} \mathrm{Gne}_{\mathrm{Ah}}$ plasmids (a single O7-repeat unit) increases slightly (twofold) their DOC resistance. However, introduction of pBAD-WecP-Wzy ${ }_{\mathrm{Ah}}+$ pGEMT-Gne ${ }_{\mathrm{Ah}}$ plasmids allow the practically recovering of the DOC resistance, since it is similar as the wildtype strain. No changes were observed when the plasmid vector alone was introduced.

These results prompted to us to purify core LPS-linked ECA fractions from several strains as indicated in the 'Materials \& methods' section. Table 2 shows that wild-type strain was able to synthesize $\mathrm{ECA}_{\mathrm{LPS}}$ fraction $(150 \mu \mathrm{g}$ per 11 of cell culture at $\mathrm{OD}_{600}=1.2$ ) detected as aminosugars after reduction and $\mathrm{HCl}$ hydrolysis $(17.5 \mu \mathrm{g}$ of mannosamine [ManN] and $19.8 \mu \mathrm{g}$ of glucosamine $[\mathrm{GlcN}]$ per 11 of culture). No such ECA fraction was obtained in $E$. coli MV501 wecA mutant. A large amount of ECA-like fraction could be detected when $E$. coli MV501 harbored plasmids pGEMT-Gne ${ }_{\mathrm{Ah}}+$ pBAD-WecP-Wzy $\left(557 \mu \mathrm{g}\right.$ per 11 of cell culture at $\left.\mathrm{OD}_{600}=1.2\right)$ with an amount of ManN $(64.0 \mu \mathrm{g})$ and GalN $(71.3 \mu \mathrm{g})$ per liter of culture.

\section{- Cross-complementation studies on E. coli wecA mutants from serotypes 0150 \& 0159}

The E. coli O7-antigen repeat unit is a pentasaccharide that contains 4-acetamido-4,6-dideoxyD-glucose (D-Qui4NAc) at its nonreducing end (Figure 1B) [40], which is an uncharacteristic sugar in $E$. coli $\mathrm{O}$-antigens [47]. For this reason, we tested other $E$. coli strains from serotypes not containing these deoxyamino monosaccharides (Figure 1C \& D).

We obtained as described in the 'Materials $\&$ methods' section in-frame wec $A$ mutants O150 $\Delta$ wec $A$ and $0159 \Delta w e c A$ from $E$. coli strains O25b-ST131 and CCUG 36899 (O150 and O159, respectively) [19]. These mutants were unable to produce O-antigen LPS as could be observed in Figures $4 \& 5$ according to their LPS profile in gels. Both mutants showed identical LPS profile as their wild-type strain O25b-ST131 or CCUG 36899 (O150 and O159, respectively) when we introduced plasmid pBAD33-WecA $\mathrm{Ec}_{\mathrm{c}}$. According to their LPS profiles on gels, neither the plasmid vector alone nor plasmid pBAD-WecP ${ }_{\mathrm{Ah}}$ or pGEMTGne $_{\text {Ah }}$ alone fully complemented the mutants under expressing conditions (+ arabinose). When we introduced $\mathrm{pBAD}-\mathrm{WecP}_{\mathrm{Ah}}$ and pGEMT$\mathrm{Gne}_{\mathrm{Ah}}$ together in the $\mathrm{O} 150 \Delta$ wec $A$ or O159 1 wec $A$ mutant, as previously described for $E$. coli MV501 (WecA), their LPS profile on gels showed two bands (one with the same motility as the mutant and the other one with a reduced motility), always when cells were grown under inducing conditions (+ arabinose; Figures 4 \& 5, Lane 3). Sugar analysis of purified LPS from these $E$. coli WecA mutants harboring pGEMT-Gne ${ }_{\mathrm{Ah}}+\mathrm{pBAD} 33-\mathrm{WecP}_{\mathrm{Ah}}$, grown under inducing conditions, showed rhamnose (Rha) in the case of O150 15 wecA + pGEMTGne $_{\mathrm{Ah}}$ and $\mathrm{pBAD} 33-\mathrm{WecP}_{\mathrm{Ah}}$ strain and fucose or galacturonic acid (Fuc and GalA, respectively) in the case of O159 wec $A+$ pGEMT-Gne $_{\mathrm{Ah}}$ and pBAD33-WecP ${ }_{A h}$ strain (Figure 3). The presence of both sugars with the LPS profile in gels suggested a single E. coli $\mathrm{O} 150$-antigen or O159-antigen 
(A)
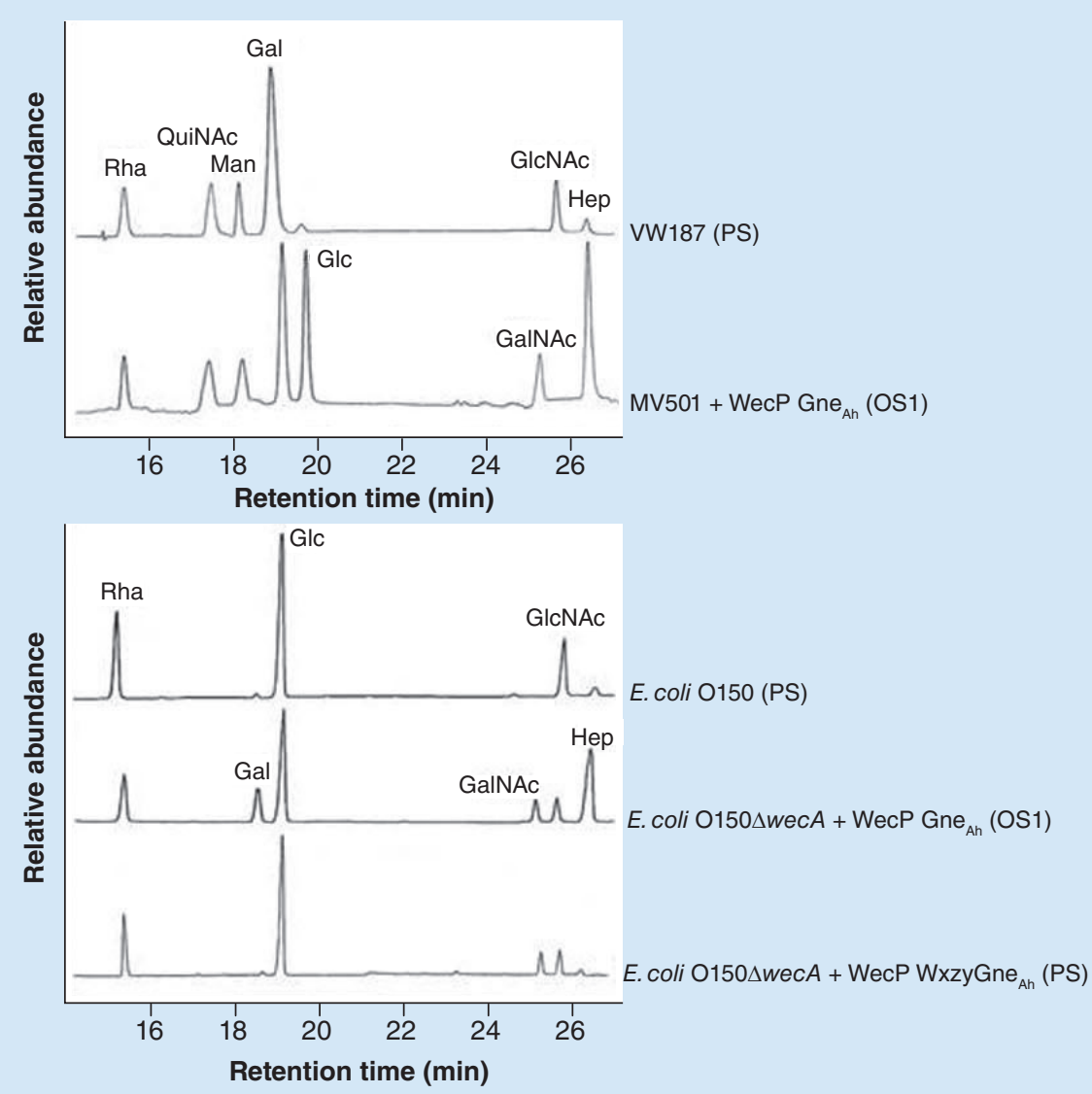

(B)

\begin{tabular}{|c|c|c|c|c|c|c|}
\hline & \multicolumn{6}{|c|}{ nmoles/mg of LPS fraction } \\
\hline OS, OS1 or Ps Fraction & GICNAC & GalNAc & Gal & Man & Rha & QuiNAc \\
\hline VW187(PS) & 198 & 0 & 588 & 196 & 192 & 184 \\
\hline MV501 + WecPGne $_{\text {An }}$ (OS1) & 0 & 125 & 380 & 122 & 118 & 115 \\
\hline \multirow[t]{2}{*}{ MV501+ WecPWzy Gne An (OS) } & 0 & 0 & 246 & 0 & 0 & 0 \\
\hline & & GIcNA & & GalNAc & Glc & Rha \\
\hline \multicolumn{2}{|l|}{\begin{tabular}{|l} 
E. coli 0150 (PS) \\
\end{tabular}} & 374 & & 0 & 740 & 561 \\
\hline \multicolumn{2}{|c|}{ E. coli $0150 \Delta w e c A+$ WecP Gne ${ }_{\text {An }}$ (OS1) } & 72 & & 85 & 390 & 248 \\
\hline \multicolumn{2}{|c|}{ E. coli $0150 \Delta w e c A+$ WecP Wzy Gne ${ }_{\text {An }}$ (PS) } & 189 & & 194 & 747 & 568 \\
\hline 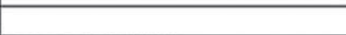 & - & \multirow{2}{*}{\multicolumn{2}{|c|}{$\frac{\text { GICNAC }}{582}$}} & GalNAc & Fuc & GalA \\
\hline \multicolumn{2}{|l|}{ E. coliO159 (PS) } & & & 0 & 578 & 285 \\
\hline \multicolumn{2}{|l|}{ E. coli $0159 \Delta$ wecA +WecP Gne (OS1) } & 134 & & 142 & 272 & 269 \\
\hline \multicolumn{2}{|c|}{ E. coli $0159 \Delta w e c A+$ WecP Wzy Gne An $_{\text {(PS) }}$} & 276 & & 287 & 562 & 498 \\
\hline
\end{tabular}

Figure 3. Gas-liquid chromatography of purified lipopolysaccharide fractions from Escherichia coli mutants and its complementations. (A) Gas-liquid chromatograms of purified lipopolysaccharide fractions (OS: core oligosaccharide fraction; OS1: core oligosaccharide with a single repeat unit; and PS, O-antigen fraction) from the E. coliswecA mutants and its complementation. (B) Monosaccharide contents of these lipopolysaccharide fractions determined by GLC. The identity of the polysaccharides is as follows: Gal: Galactose; GalA: D-Galacturonic acid; GalNAc: N-D-acetylgalactosamine; GlcNAc: N-acetyl-D-glucosamine; D-Glc: D-glucose; Fuc: D-Fucose; Hep: L-D-heptoses; Man: D-Mannose; QuiNAc: N-acetyl-D-quinovosamine; Rha: L-Rhamnose. 
Table 2. Sensitivity to deoxicholate measured as minimum inhibitory concentration and enterobacterial common antigen production in Escherichia coli VW187 (serotype 07) and mutants.

\begin{tabular}{|c|c|c|}
\hline Strain & DOC (MIC) & ECA production $^{\dagger}$ \\
\hline Wild type & $1 \%$ & $+(150 \mu \mathrm{g} / \mathrm{l})$ \\
\hline MV501 (wecA mutant) & $0.15 \%$ & $-(<1 \mu g / l)$ \\
\hline MV501 + pGEMT-Gne ${ }_{A h}+$ pBAD33-WecP ${ }_{\text {Ah }}$ & $0.3 \%$ & NT \\
\hline MV501 + pGEMT-Gne ${ }_{A h}$ & $0.15 \%$ & NT \\
\hline MV501 + pGEMT-Gne ${ }_{\text {Ah }}+$ pBAD33-WecP-Wzy ${ }_{A h}$ & $0.8 \%$ & $+++(557 \mu \mathrm{g} / \mathrm{l})$ \\
\hline MV501 + pBAD33-WecP-Wzy ${ }_{A h}$ & $0.15 \%$ & NT \\
\hline \multicolumn{3}{|c|}{$\begin{array}{l}\text { 'The strains carrying pBAD plasmids were grown under inducing conditions (+ arabinose). } \\
+: \text { Positive; -: Negative; +++: Highly positive. The numeric value corresponds to per } 1 \text { I of cells culture at } \mathrm{OD}_{600}=1.2 \text { as described in } \\
\text { the 'Materials \& methods' section. } \\
\text { DOC: Deoxycholate; ECA: Enterobacterial common antigen; MIC: Minimum inhibitory concentration; NT: Not tested. }\end{array}$} \\
\hline
\end{tabular}

LPS repeat unit [41,42]. LPS isolated from E. coli WecA mutants in both cases lack Rha and Fuc/ GalA, two monosaccharides absent in the different E. coli LPS cores described [41,42]. However, when both mutants carry the following plasmids: pGEMT-Gne ${ }_{\mathrm{Ah}}+$ pBAD-WecP-Wzy ${ }_{\mathrm{Ah}}$, altogether but not separately, and were grown under inducing conditions a full recovery of their specific O-antigen LPS, judged by their LPS profile in gels is achieved (Figures 4 \& 5, Lane 4). When we purified the LPS from these $E$. coli WecA mutants harboring pGEMT-Gne ${ }_{\mathrm{Ah}}+\mathrm{pBAD}-\mathrm{WecP}-\mathrm{Wzy}_{\mathrm{Ah}}$ we identified $\mathrm{Rha}$ in the case of O150 strain and Fuc/GalA in the case of O159 strain by sugar analyses (Figure 3) $[41,42]$. In both cases the amount of Rha or Fuc were several times higher (ratio Rha/ Hep or Fuc/Hep) than previously indicated for the same mutants harboring only pGEMT-Gne ${ }_{\mathrm{Ah}}$ and pBAD33-WecP ${ }_{\text {Ah }}$ plasmids. These results, in addition to the LPS profiles in SDS gels, suggested the presence of several $\mathrm{O}$-antigen repetition units when $\mathrm{Wzy}_{\mathrm{Ah}}$ is added. No changes in their LPS profile were observed when both WecA mutants harbored pBAD33-WecP-Wzy ${ }_{\mathrm{Ah}}$ without pGEMT-Gne $_{\mathrm{Ah}}$ (Figures 4 \& 5, Lane 5).

\section{- Topology of O-antigen polymerases (Wzy)}

The $\mathrm{O}$-antigen polymerases are generally extremely hydrophobic and have $\geq 11$ putative transmembrane domains [48-50]. However, topology maps predicted by TMMTOP v2.0 [29] show that, except for the E. coli $\mathrm{DH} 5 \alpha \mathrm{Wzy}-\mathrm{ECA}\left(\mathrm{WzyE}_{\mathrm{Ec}}\right.$ ) which contains 13 putative transmembrane domains, the remaining $\mathrm{O}$-antigen polymerases of strains studied contains a lower number of transmembrane domains (TMs): ten TMs are present in E. coli Wzy-O150, nine TMs in E. coli Wzy-O159 and the $A$. hydrophila Wzy-O34 $\left(\mathrm{Wzy}_{\mathrm{Ah}}\right)$ and seven TMs in E. coli Wzy-O7 (Figure 6). Furthermore, the averages of hydropathicity (GRAVY) of the $\mathrm{O}$-antigen polymerases $\mathrm{WzyE}_{\mathrm{Ec}}, \mathrm{Wzy}_{\mathrm{Ah}}$ and $\mathrm{Wzy}$ O159 are lower (0.81, 0.82 and 0.89 , respectively) than the estimated for Wzy-O150 (1.04) and higher than the estimated for Wzy-O7 (0.66).

The E. coli DH5 $\alpha$ Wzy-ECA contains six periplasmic loops (PLs), being PL2 and 5 the larger ones (each with 22 amino acid residues). The E. coli Wzy-O150, Wzy-O159 and the A. hydrophila Wzy-O34 are predicted to have four PLs, being PL1, 2 and 3 the larger ones in E. coli Wzy-O150 and Wzy-O159 (30, 26 and 30 amino acid residues; and 43, 26, 40 amino acid residues, respectively); and PL 3 and 4 the larger ones in A. hydrophila Wzy-O34 (44 and 88 amino acids). The $E$. coli Wzy-O7 only contains three PLs being PL 3 the larger with 146 amino acids (Figure 6). As described in Shigella flexneri and Pseudomonas aeruginosa [49,50], A. hydrophila Wzy-O34 shows two RX $\mathrm{n}_{\mathrm{n}}$ motifs in PL3 and PL4 (RX $\mathrm{RX}_{15}$ and $\mathrm{RX}_{17} \mathrm{G}$, respectively). However, E. coli Wzy-ECA and Wzy-O150 contain a motif $\mathrm{RX}_{\mathrm{n}} \mathrm{G}$ in PL2 $\left(\mathrm{RX}_{13} \mathrm{G}\right.$ and $\mathrm{RX}_{17} \mathrm{G}$, respectively) and a motif $\mathrm{HX}_{\mathrm{n}} \mathrm{G}$ in PL5 and PL1 ( $\mathrm{HX}_{10} \mathrm{G}$ and $\mathrm{HX}_{12} \mathrm{G}$, respectively). In contrast, E. coli WzyO159 contains three RX $G$ motifs: a $R_{15} G$ motif on PL1 and 3; and a $\mathrm{RX}_{17} \mathrm{G}$ in PL2. The E. coli Wzy-O7 contains three $\mathrm{RX}_{\mathrm{n}} \mathrm{G}$ motifs and all of them localized in PL3 $\left(\mathrm{RX}_{10} \mathrm{G}, \mathrm{RX}_{17} \mathrm{G}\right.$ and $\mathrm{RX}_{22} \mathrm{G}$ ) (Figure 6).

\section{Discussion}

Many E. coli O-antigen repeat units have $N$-acetylglucosamine-phosphate (GlcNAc-1-P) as first sugar, which is transferred to the Und-P by WecA, to give Und-PP-linked GlcNAc [5]. Other E. coli $\mathrm{O}$-antigens have $N$-acetylgalactosaminephosphate (GalNAc-1-P) at the reducing end, and just two have $N$-acetylfucosamine-phosphate 
(FucNAc-1-P) [47]. In E. coli O157, GalNAcUndP is synthesized from GlcNAc-UndP by an epimerase capable of interconverting GlcNAc-PP-Und and GalNAc-P-P-Und [51]. However, in A. hydrophila AH-3 the GalNAc-1-P is synthetized by the Gne epimerase, which have two enzymatic activities: UPD-Gal and UDPGalNAc 4-epimerimerase. Gne is responsible for the conversion of UDPGlcNAc to UDPGalNAc and the transfer of GalNAc-1-P to Und-P to give Und-PP-linked

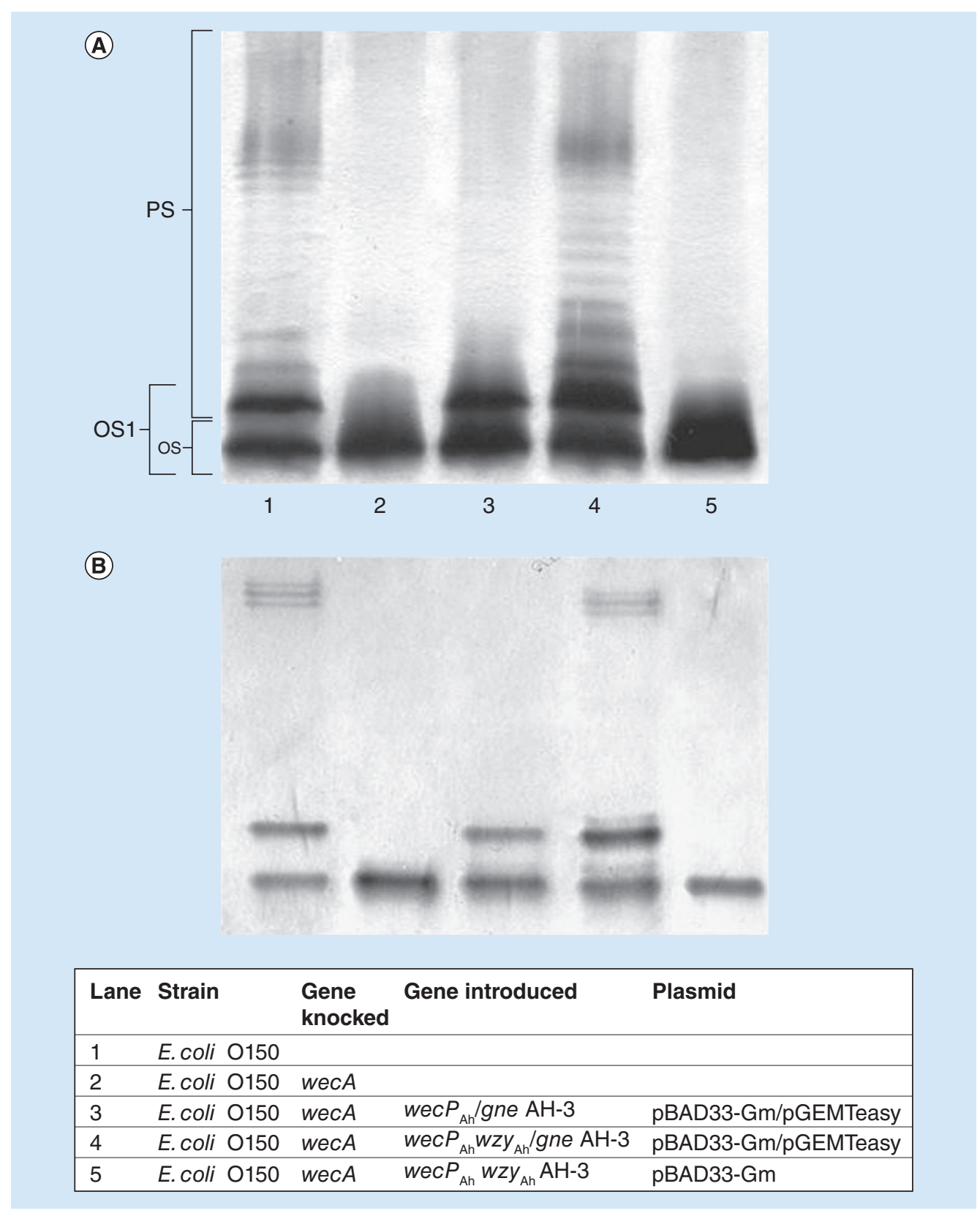

Figure 4. Polyacrylamide gels showing the migration of lipopolysaccharides from Escherichia coli $0150 \Delta$ wecA mutant and its complementation. The lipopolysaccharide (LPS) samples were separated on SDS-PAGE (A) and SDS-Tricine-PAGE (B) and visualized by silver staining. Shown are LPS samples

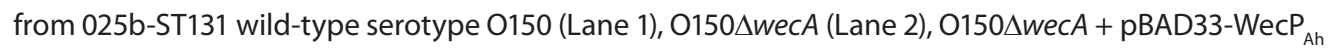
and pGEMT-Gne (Lane 3), O150 $\Delta w e c A+$ pBAD-WecP-Wzy ${ }_{A h}$ and pGEMT-Gne (Lane 4) and O150 $\Delta w e c A+$ PBAD-WecP-Wzy ${ }_{A h}$ (Lane 5). LPS fractions: OS: core oligosaccharide fraction; OS1: core oligosaccharide with a single repeat unit; and PS: $\mathrm{O}$-antigen fraction. All the strains harboring pBAD plasmids were grown under induced conditions (+ arabinose) as indicated in the 'Materials \& methods' section. 


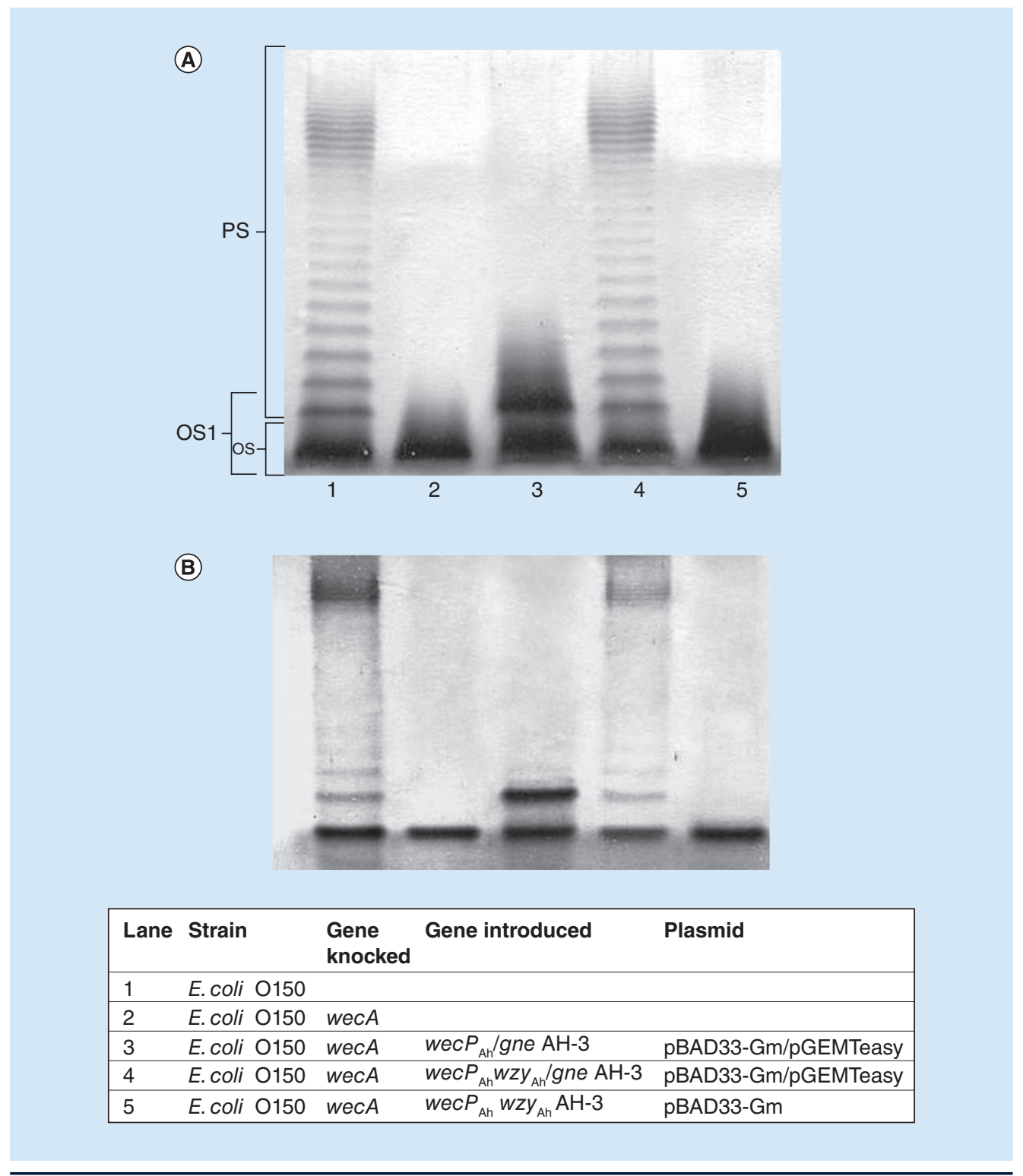

Figure 5. Polyacrylamide gels showing the migration of lipopolysaccharide from Escherichia coli 0159 $\Delta$ wecA mutant and its complementation. The LPS samples were separated on SDS-PAGE (A) and SDS-Tricine-PAGE (B) and visualized by silver staining. Shown are lipopolysaccharide samples from

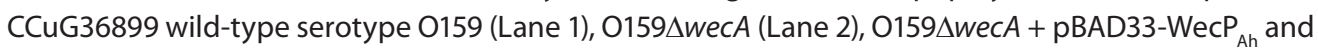

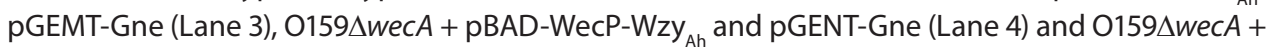
pBAD-WecP-Wzy ${ }_{A h}$ (Lane 5). LPS fractions: OS: core oligosaccharide fraction; OS1: core oligosaccharide with a single repeat unit; and PS: $\mathrm{O}$-antigen fraction. All the strains harboring pBAD plasmids were grown under induced conditions (+ arabinose) as indicated in the 'Materials \& methods' section.

GalNAc, is performed by the initial transferase WecP [7].

The E. coli O7-antigen repeat unit is a pentasaccharide of D-GlcNAc, galactose (Gal), D-mannose (Man), Rha and 4-acetamido-4,6dideoxy-D-glucose (D-Qui4NAc) [40] (Figure 1B). The abolishment of WecA, which transfers the GlcNAc-1-P to Und-P [5], leads to the absence of
O7-antigen repeat unit (Figure 2, Lane 2). In this $E$. coli $\mathrm{O} 7$ background, the $A$. hydrophila WecP, which transfers the GalNAc-1-P to Und-P [7], is unable to restore the LPS profile (Figure 2, Lane 6). Escherichia coli $\mathrm{O} 7$ lacks Gne epimerase and, only when this gene is present and expressed this strain is able to obtain the GalNAc required for the WecP activity [22]. The E. coli $\mathrm{O} 7$ wecA mutant 
carrying gne $+w e c P$ in expressing vectors reveals an LPS profile on gels with two bands (Figure 2, Lane 3), characteristic of an LPS-core with a single $\mathrm{O}$-antigen repeat, and the chemical data indicate the presence of GalNAc (Figure 3). These data suggest that GalNAc is the first sugar of the repeat unit and E. coli Wzx-O7 is able to translocate it, but E.coli Wzy-O7 does not have the ability to polymerize the $\mathrm{O}$-antigen repeat units. Surprisingly, E. coli $\mathrm{O} 7$ wecA mutant with gne + $w e c P-w z y_{\mathrm{Ah}}$ loses the characteristic LPS double band and shows a single band (Figure 2, Lane 4) and production of ECA-like molecules. The chemical data indicate the lack of the O7-antigen single repeat when the wecA mutant carries gne + $w e c P-w z y_{\mathrm{Ah}}$ (Figure 3). By sensitivity to DOC and chemical methods, we show that ECA-like molecules were produced when the wecA mutant carries gne $+w e c P-w z y_{\mathrm{Ah}}$, which were not previously found when the wecA mutant carried only gne + wecP (Table 2). Similar results were seen in the E. coli $\mathrm{O} 121$ background whose $\mathrm{O}$-antigen repeat unit is a tetrasaccharide containing GlcNAc at the reducing end and 4-( $N$-acetylglycyl) amino-4,6-dideoxy- $D$-glucose, $D$-Qui $4 \mathrm{~N}$ ( $N$-acetyl-glycyl), at the nonreducing end (data not shown).

The repeat units of the E. coli O150 and O159antigens are a hexasaccharide and a pentasaccharide, respectively (Figure $1 C \& D$ ), which contain $\beta$-D-GlcNAc at both the reducing and nonreducing ends. As in $E$. coli $\mathrm{O} 7$, the wecA mutation abolishes formation of the $\mathrm{O}$-antigen repeat unit

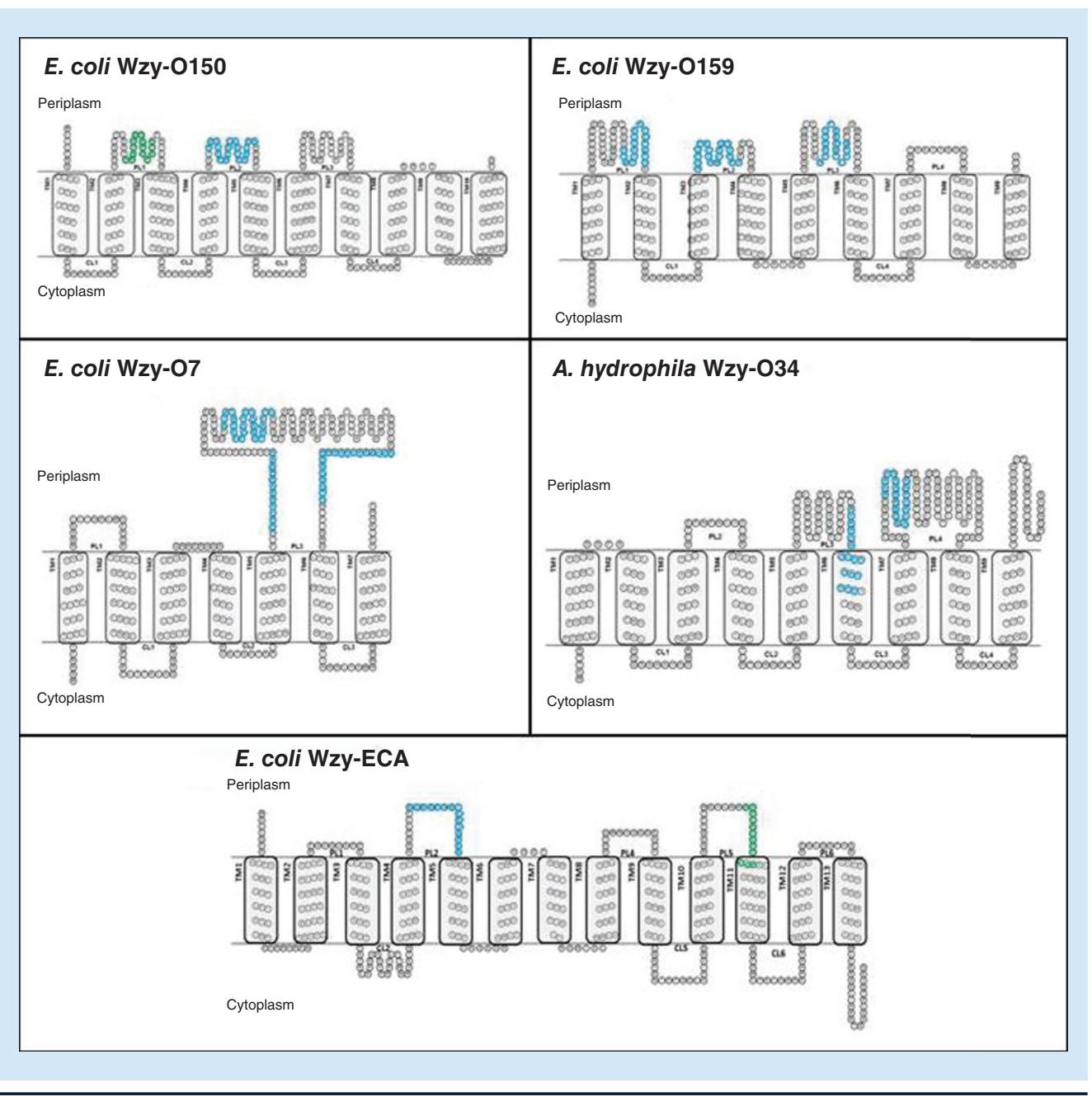

Figure 6. Topological comparison of O-antigen polymerases. Theoretical topology map of the $\mathrm{O}$-antigen polymerases in Escherichia coli $\mathrm{Wzy}_{\mathrm{Ec}}$ (enterobacterial common antigen Wzy polymerase), Wzy-O150, Wzy-O159 and Wzy-O7; and A. hydrophila Wzy-O34 (Wzy $\left.{ }_{\mathrm{Ah}}\right)$. Topology maps were predicted by TMMTOP v2.0 [29]. PL1-n, number periplasmic loops; CL1-n, number cytoplasmic loops; and TM1-n, number transmembrane domains. Blue circles show $\mathrm{RX}_{n} \mathrm{G}$ motifs and green circles $\mathrm{HX}_{\mathrm{n}} \mathrm{G}$ motifs. 
(Figures 4 \& 5, Lane 2) and $A$. hydrophila WecP is unable to perform cross-complementation. However, when $A$. hydrophila gne + wecP were introduced together in the $E$. coli wec $A$ mutants, a characteristic LPS profile with two bands was observed (Figures 4 \& 5, Lane 3), and the chemical data indicated the presence of GalNAc in the $\mathrm{O}$-antigen repeat unit (Figure 3 ). In contrast to what was observed in E. coli O7, the concerted action of $A$. hydrophila $\mathrm{Wzy}_{\mathrm{Ah}}$, with the corresponding $\mathrm{WecP}$ in the presence of Gne is able to polymerize the corresponding $\mathrm{O} 150$ or O159-antigen units (Figures 4 \& 5, Lane 4). The chemical data obtained allow us to indicate that the $\mathrm{O}$-antigen LPS correspond to E. coli serotypes O150 or O159-like (Figure 3). Therefore, in E. coli O150 or O159 backgrounds, the results are similar to the ones obtained in A. hydrophila $\mathrm{O} 34$ [24], since the AH-3 4 wecP mutant with the $E$. coli WecA, from the ECA cluster, is able to elongate the chain with a single $\mathrm{O}$-antigen unit, but polymerization of the O34-antigen is only produced when E. coli WecA-WzyE $\mathrm{Ec}_{\mathrm{Ec}}$ (both from the ECA cluster) are present together. Furthermore, plasmid pBAD$\mathrm{WzyE}_{\mathrm{Ec}_{\mathrm{c}}}$ [carrying the E. coli ECA-Wzy] is unable to restore the $\mathrm{Wzy}_{\mathrm{Ah}}$ function and polymerize the O:34-antigen when was introduced in the $A$. hydrophila AH-3 $\mathrm{Wzy}_{\mathrm{Ah}}$ mutant (A405-wzy) and expressed with arabinose.

Figure 1 shows the chemical structure of the A. hydrophila $\mathrm{O} 34$-antigen LPS, E. coli O7, O150, O159-antigen LPSs and ECA from E. coli. For all these $\mathrm{O}$-antigen polysaccharides the initial sugar linked to the Und-P is a HexNAc. The E. coli O7-antigen LPS repeating units, contain at the distal position (nonreducing end) 4-acetamido4,6-dideoxy- $D$-glucose ( $D$-Qui4NAc), which is an unusual sugar for prokaryote polysaccharides. We suggest that $A$. hydrophila Wzy is unable to recognize D-Qui4NAc at the nonreducing end of the $\mathrm{O} 7$ repeating unit to form a glycosidic bond with the reducing end of the growing chain. However, it is able to recognize 4-acetamido-4, 6-dideoxy-D-galactose (D-Fuc4NAc) and produces ECA in the E. coli $\mathrm{O} 7$ background (Figure 1E), as shown the DOC resistance and the ECA amount in the E.coli MV501 with pBAD-WecP-Wzy ${ }_{\mathrm{Ah}}+$ pGEMT-Gne ${ }_{\mathrm{Ah}}$ plasmids (Table 2). Nevertheless, it seems that $\mathrm{O}$-antigen LPS is always the biosynthetic preference among the different Gram-negative bacterial surface polysaccharides in this background.

The O-antigen polymerases show high level of amino acid sequence diversity and absence of conserved regions, which has related to differences in the composition and structure of translocated $\mathrm{O}$-antigen repeat units. Although Wzy mediates the formation of a glycosidic linkage between the nonreducing end of the new repeat subunit and the reducing end of the growing chain, it displays no homology to other known glycosyltransferases. The $\mathrm{O}$-antigen polymerases are integral membrane proteins with 11-13 transmembrane domains, responsible for the polymerization of $\mathrm{O}$-antigens, capsules and other cell surface polysaccharides [52,53]. However, topology maps predicted by TMMTOP v2.0 [29] show that $E$. coli Wzy-O7, Wzy-O150 and Wzy-O159, as well as $A$. hydrophila Wzy-O34 have lower number of transmembrane domains (TMs) (Figure 6). The E. coli Wzy-O7 contains the lower number of TMs and average of hydropathicity. Two larger periplasmic loops essential for the catalytic activity of Wzy have been described in Pseudomonas aeruginosa, Shigella flexneri and Francisella tularensis (PL3 and 5) [48-50]. We also found two larger periplasmic loops in the E. coli Wzy-ECA and A. hydrophila Wzy-O34 (PL 2 and 5; and PL3 and 4, respectively). However, the E. coli Wzy-O150 and WzyO159 contain three large periplasmic loops, and E. coli Wzy-O7 only contains one (Figure 6). It has been hypothesized that the periplasmic domain of the $\mathrm{O}$-antigen polymerase is involved in bonding the $\mathrm{O}$-antigen repeat units and polymerizing them into the O-antigen chain [54]. In S. flexneri and $P$. aeruginosa Wzy, the two larger periplasmic loops contain $\mathrm{RX}_{\mathrm{n}} \mathrm{G}$ motifs involved in their polymerase activity $\left(\mathrm{RX}_{15} \mathrm{G}\right.$ and $\mathrm{RX}_{10} \mathrm{G}$, respectively) [49,50]. A hydrophila Wzy-O34 also contains $\mathrm{RX}_{\mathrm{n}} \mathrm{G}$ motifs in PL3 and PL4, although with different lengths ( $\mathrm{RX}_{15} \mathrm{G}$ and $\mathrm{RX}_{17} \mathrm{G}$, respectively). However, E. coli Wzy-ECA and Wzy-O150 contain a motif RX $\mathrm{G}$ and a motif $\mathrm{HX}_{\mathrm{n}} \mathrm{G}$ : $\mathrm{RX}_{13} \mathrm{G}$ and $\mathrm{HX}_{10} \mathrm{G}$ in Wzy-ECA and $\mathrm{HX}_{12} \mathrm{G}_{\text {and }} \mathrm{RX}_{17} \mathrm{G}$ in Wzy-O150. Escherichia coli Wzy-O159 shows three RX $\mathrm{G}$ motifs $\left(\mathrm{RX}_{15} \mathrm{G}\right.$ in PL1 and 3, and $\mathrm{RX}_{17} \mathrm{G}$ in PL2), as well as E. coli Wzy-O7, although all of them are localized in PL3 $\left(\mathrm{RX}_{10} \mathrm{G}, \mathrm{RX}_{17} \mathrm{G}\right.$ and $\mathrm{RX}_{22} \mathrm{G}$ ) (Figure 6). All these data indicate some diversity among the different strains in Wzy polymerases, being E. coli Wzy-O7 by large the most different one.

Previous work demonstrated that Wzx proteins from O-antigen systems that use GlcNAc or GalNAc as initial sugar of the repeat unit were able to translocate $\mathrm{O}$-antigen repeat units whose initial sugar was any of them [55], as well as Salmonella Wzy is able to discriminate between 
O-antigen units possessing identical sugars but different internal sugar linkage [56]. However, we demonstrate that E. coli $\mathrm{WzyE}_{\mathrm{Ec}}$ (ECA Wzy polymerase) is able to polymerize the $A$. hydrophila $\mathrm{O} 34$ antigen although requiring the concerted action of WecA [24]. Therefore, the Wzx/Wzy $\mathrm{O}$-antigen export pathway is not strain dependent, as it was suggested in some cases, it depends on the UDP-HexNAc:polyprenol-P HexNAc-1-P transferase (WecA/P) and their compatibility with the corresponding Wzy (O-antigen polymerase). Given the WecA mutation abolishes the transfer of D-GlcNAc to the Und-P and do not modify the terminal sugar of the repeat unit, the introduction of $A$. hydrophila Gne + WecP only changes the sugar at the reducing end and allows translocation of the repeat unit. However, the E. coli Wzy polymerase is unable to polymerize the repeat unit. We suggested that, in the $\mathrm{W}_{z x} / \mathrm{Wzy}_{z}$-dependent $\mathrm{O}$-antigen export and assembly pathway, the activity of the $\mathrm{O}$-antigen polymerase (Wzy) is dependent on the first sugar of the $\mathrm{O}$-antigen repeat unit to produce the O-antigen polymerization. Furthermore, it needs the concerted action of the corresponding enzyme transferring the initial HexNAc to undecaprenol phosphate (UDP-HexNAc: polyprenol-P HexNAc-1-P transferase) to transfer the growing units from the Und-P to the nonreducing end of the new $\mathrm{O}$-subunit. Our results support that some Wzy are permissive with the sugar at the nonreducing end of the $\mathrm{O}$-antigen repeat unit, as observed in Aeromonas hydrophila Wzy-O34.

\section{Conclusion}

The Wzx/Wzy-dependent O-antigen export and assembly pathway is not as highly specific as it has been suggested previously, since the ability to polymerize the $\mathrm{O}$-antigen depends on the compatibility between the UDPHexNAc:polyprenol-P HexNAc-1-P transferase and the O-antigen polymerase (Wzy). Therefore, the system is able to assemble $\mathrm{O}$-antigen units possessing different monosaccharide chemical structures when provided on a compatible first monosaccharide. Furthermore, some $\mathrm{O}$-antigen polymerases are also permissive with the sugar at the nonreducing end of the $\mathrm{O}$-antigen repeat unit.

\section{Acknowledgements}

The authors would like to thank Maite Polo for her technical assistance and the Servicios Cientifico-Técnicos from University of Barcelona.

\section{Financial \& competing interests disclosure}

This work was supported by Plan Nacional de $I+D+i$ (Ministerio de Educación, Ciencia y Deporte and Ministerio de Sanidad, Spain) and from Generalitat de Catalunya (Centre de Referència en Biotecnologia). The authors have no other relevant affliations or financial involvement with any organization or entity with a financial interest in or financial conflict with the subject matter or materials discussed in the manuscript apart from those disclosed.

No writing assistance was utilized in the production of this manuscript.

\section{Open access}

This work is licensed under the AttributionNonCommercial-NoDerivatives 4.0 Unported License. To view a copy of this license, visit http://creativecommons.org/ licenses/by-nc-nd/4.0/

\section{EXECUTIVE SUMMARY}

- The Aeromonas hydrophila WecP, which transfers the GaINAc-1-P to Und-P, is unable to restore the lipopolysaccharide profile of Escherichia coli O7, 0150 and 0159 in an E. coli wecA background.

- E. coli O7, 0150 and 0159 wecA mutants show an LPS-core with a single O-antigen repetition when A. hydrophila WecP + Gne, a UDP-GalNAc 4-epimerase enzyme responsible for the conversion of UDP-GlcNAc to UDP-GalNAc, were transferred.

- The O-antigen polymerases (Wzy) from E.coli O7, $\mathrm{O} 150$ and $\mathrm{O} 159$ are unable to polymerize O-antigen repeat units in which the first sugar is GalNAc.

- E. coli $\mathrm{O} 7$ wecA mutant with A. hydrophila WecP + Gne + Wzy loses the single O-antigen repetition and produces ECAlike molecules.

- E. coli $\mathrm{O} 150$ and $\mathrm{O} 159$ wecA mutants with A. hydrophila WecP + Gne + Wzy are able to polymerize the corresponding O150 or O159-antigen units.

- Analyses of O-antigen polymerases show some diversity among the different strains, being E. coli Wzy-O7 the most different one. 


\section{References}

1 Aquilini E, Tomás JM. Lipopolysaccharides (endotoxins). In: Reference Module in Biomedical Sciences. Elsevier. doi:10.1016/ B978-0-12-801238-3.07799-0 (2015) (Epub ahead of print).

2 Nikaido H. Outer membrane. In: Escherichia coli and Salmonella: Cellular and Molecular Biology. Neidhardt FC, Curtiss R, Ingraham JL et al. (Eds). ASM Press, USA, 29-47 (1996).

3 Feng L, Senchenkova SN, Yang J et al. Structural and genetic characterization of the Shigella boydii type $13 \mathrm{O}$ antigen. J. Bacteriol. 186(2), 383-392 (2004).

4 Hug I, Feldman MF. Analogies and homologies in lipopolysaccharide and glycoprotein biosynthesis in bacteria. Glycobiology 21(2), 138-151 (2011).

5 Samuel G, Reeves P. Biosynthesis of $\mathrm{O}$-antigens: genes and pathways involved in nucleotide sugar precursor synthesis and O-antigen assembly. Carbohydr. Res. 338(23), 2503-2519 (2003).

6 Jiménez N, Canals R, Saló MT, Vilches $S$, Merino S, Tomás JM. The Aeromonas hydrophila $w b_{\mathrm{O} 34}^{*}$ gene cluster: genetics and temperature regulation. J. Bacteriol. 190(12), 4198-4209 (2008).

7 Merino S, Jiménez N, Molero R, Bouamama L, Regué M, Tomás JM. A UDP-HexNAc: polyprenol-P GalNAc-1-P transferase (WecP) representing a new subgroup of this enzyme family. J. Bacteriol. 193(8), 1943-1952 (2011).

8 Liu D, Cole RA, Reeves PR. An O-antigen processing function for Wzx (RfbX): a promising candidate for O-unit flippase. J. Bacteriol. 178(7), 2102-2107 (1996).

9 Islam ST, Lam JS. Wzx flippase-mediated membrane translocation of sugar polymer precursors in bacteria. Environ. Microbiol. 15(4), 1001-1015 (2013).

10 Collins LV, Hackett J. Molecular cloning, characterization, and nucleotide sequence of the rfc gene, which encodes an $\mathrm{O}$-antigen polymerase of Salmonella typhimurium. J. Bacteriol. 173(8), 2521-2529 (1991).

11 Kalynych S, Valvano MA, Cygler M. Polysaccharide co-polymerases: the enigmatic conductors of the $\mathrm{O}$-antigen assembly orchestra. Protein Eng. Des. Sel. 25(11), 797-802 (2012).

12 Abeyrathne P, Daniels C, Poon KK, Matewish MJ, Lam J. Functional characterization of WaaL, a ligase associated with linking $\mathrm{O}$-antigen polysaccharide to the core of Pseudomonas aeruginosa lipopolysaccharide. J. Bacteriol. 187(9), 3002-3012 (2005).
13 Silhavy TJ, Kahne D, Walker S. The bacterial cell envelope. Cold Spring Harb. Perspect. Biol. 2(5), a000414 (2010).

14 Islam ST, Lam JS. Synthesis of bacterial polysaccharides via the Wzx/Wzy-dependent pathway. Can. J. Microbiol. 60(11), 697-716 (2014).

15 Robbins P W, Bray D, Dankert BM, Wright A. Direction of chain growth in polysaccharide synthesis. Science 158(3808), 1536-1542 (1967).

16 Papadopoulos M, Morona R. Mutagenesis and chemical cross-linking suggest that Wzz dimer stability and oligomerization affect lipopolysaccharide $\mathrm{O}$-antigen modal chain length control. J. Bacteriol. 192(13), 3385-3393 (2010).

17 Hanahan D. Studies on transformation of Escherichia coli with plasmids. J. Mol. Biol. 166(4), 557-580 (1983).

18 Alexander DC, Valvano MA. Role of the rfe gene in the biosynthesis of the Escherichia coli O7-specific lipopolysaccharide and other $\mathrm{O}$-specific polysaccharides containing $\mathrm{N}$-acetylglucosamine. J. Bacteriol. 176(22), 7079-7084 (1994).

19 Kaufmann M, Zweifel C, Blanco M et al. Escherichia coli $\mathrm{O} 157$ and non-O157 Shiga toxin-producing Escherichia coli in fecal samples of finished pigs at slaughter in Switzerland. J. Food Prot. 69(2), 260-266 (2006).

20 Aguilar A, Merino S, Rubires X, Tomás JM. The influence of osmolarity on lipopolysaccharide and virulence of Aeromonas hydrophila serotype $\mathrm{O}: 34$ strains grown at $37^{\circ} \mathrm{C}$. Infect. Immun. 65(4), 1245-1250 (1997).

21 Link AJ, Phillips D, Church GM. Methods for generating precise deletions and insertions in the genome of wild-type Escherichia coli: application to open reading frame characterization. J. Bacteriol. 179(20), 6228-6237 (1997).

22 Canals R, Jiménez N, Vilches $S$, Regué $M$, Merino S, Tomás JM. The UDP $N$-acetylgalactosamine 4-epimerase gene is essential for mesophilic Aeromonas hydrophila serotype O34 virulence. Infect. Immun. 74(1), 537-548 (2006).

23 Jiménez N, Vilches S, Lacasta A, Regué M, Merino S, Tomás JM. A bifunctional enzyme in a single gene catalyzes the incorporation of GlcN into the Aeromonas core LPS. J. Biol. Chem. 284(48), 32995-33005 (2009).

24 Merino S, Gonzalez V, Tomás JM. The polymerization of Aeromonas hydrophila AH-3 $\mathrm{O}$-antigen LPS: concerted action of WecP and Wzy. PLoS ONE 10(7), e0131905 (2015).
25 Sambrook J, Fritsch EF, Maniatis T. Molecular Cloning: A Laboratory Manual, 2nd Edition. Cold Spring Harbor Laboratory, Cold Spring Harbor, NY, USA (1989).

26 Sanger F, Nicklen S, Coulson AR. DNA sequencing with chain-terminating inhibitors. Proc. Natl Acad. Sci. USA 74(12), 5463-5467 (1977).

27 Altschul FS, Madden TL, Schäffer AA et al. Gapped BLAST and PSI-BLAST: a new generation of protein database search programs. Nucleic Acid Res. 25(17), 3389-3402 (1997).

28 Bateman A, Birney E, Cerruti L et al. The Pfam protein families database. Nucleic Acids Res. 30(1), 276-280 (2002).

29 Tusnday GE, Simon I. The HMMTOP transmembrane topology prediction server. Bioinformatics 17(9), 849-850 (2001).

30 Izquierdo L, Coderch N, Piqué N et al. The Klebsiella pneumoniae wabG gene: their role in the biosynthesis of the core lipopolysaccharide and virulence. J. Bacteriol. 185(24), 7213-7221 (2003).

31 Guzman LM, Belin D, Carson MJ, Beckwith $\mathrm{J}$. Tight regulation, modulation, and high-level expression by vectors containing the arabinose PBAD promoter. J. Bacteriol. 177(14), 4121-4130 (1995).

32 Galanos C, Lüderitz O, Westphal O. A new method for the extraction of $\mathrm{R}$ lipopolysaccharides. Eur. J. Biochem. 9(2), 245-249 (1969)

33 Westphal O, Jann K. Bacterial lipopolysaccharide extraction with phenol-water and further application of the procedure. Methods Carbohydr. Chem. 5, 83-91 (1965).

34 Hitchcock PJ, Brown TM. Morphological heterogeneity among Salmonella lipopolysaccharide chemotypes in silver-stained polyacrylamide gels. J. Bacteriol. 154(1), 269-277 (1983).

35 Tsai CM, Frasch CE. A sensitive silver stain for detecting lipopolysaccharides in polyacrylamide gels. Anal. Biochem. 119(1), 115-119 (1982).

36 Mannel D, Mayer H. Isolation and chemical characterization of the enterobacterial common antigen. Eur. J. Biochem. 86(2), 361-370 (1978).

37 Dankert M, Wright A, Kelly WS, Robbins PW. Isolation, purification and properties of the lipid-linked intermediates of $\mathrm{O}$ antigen biosynthesis. Arch. Biochem. Biophys. 116(1), 425-435 (1966).

38 Brown DH. Preparation and analysis of D-Glucosamine-6-phopshate. Methods Enzymol. 3, 158-162 (1957). 
39 Knirel YA, Shaskov AS, Senchenkova SN, Merino S, Tomás JM. Structure of the O-polysaccharide of Aeromonas hydrophila $\mathrm{O} 34$ : a case of random $\mathrm{O}$-acetylation of 6-deoxy-L-talose. Carbohydr. Res. 337(15), 1381-1386 (2002).

40 L'vov VL, Shashkov AS, Dmitriev BA, Kochetkov NK, Jann B, Jann K. Structural studies of the 0 -specific sidechain of the lipopolysaccharide from Escherichia coli 07. Carbohydr. Res. 126(2), 249-259 (1984).

41 Perepelov AV, Han W, Senchenkova SN et al. Structure of the O-polysaccharide of Escherichia coli O150 containing 2-acetamido-4-O-[(S)-1-carboxyethyl]-2-deoxy-dglucose. Carbohydr. Res. 342(3-4), 648-652 (2007).

42 Rosen J, Robobi A, Nyholm PG. The conformations of the O-specific polysaccharides of Shigella dysenteriae type 4 and Escherichia coli $\mathrm{O} 159$ studied with molecular mechanics (MM3) filtered systematic search. Carbohydr. Res. 339(5), 961-966 (2004).

43 Valvano MA. Export of O-specific lipopolysaccharide. Front. Biosci. 8, 452-471 (2003).

44 Wang Y, Xu Y, Perepelov AV et al. Biochemical characterization of dTDP-D-Qui4N and dTDP-D-Qui4NAc biosynthetic pathways in Shigella dysenteriae Type 7 and Escherichia coli O7. J. Bacteriol. 189(23), 8626-8635 (2007).

45 Rahman A, Barr K, Rick PD. Identification of the structural gene for the TDP-Fuc 4 NAc: lipid II Fuc4NAc transferase involved in synthesis of Enterobacterial common antigen in Escherichia coli K-12. J. Bacteriol. 183(22), 6509-6516 (2001).

46 Ramos-Morales F, Prieto AI, Beuzón CR, Holden DW, Casadesús J. Role for Salmonella enterica Enterobacterial common antigen in bile resistance and virulence. J. Bacteriol. 185(17), 5328-5332 (2003).

47 Stenutz R, Weintraub A, Widmalm G. The structures of Escherichia coli O-polysaccharide antigens. FEMS Microbiol Rev. 30(3), 382-403 (2006).

48 Kim T, Sebastian S, Pinkham JT, Ross RA, Blalock LT, Kasper DL. Characterization of the O-antigen polymerase (Wzy) of Francisella tularensis. J. Biol. Chem. 285(36), 27839-27849 (2010).

49 Islam ST, Taylor VL, Qi M, Lam JS. Membrane topology mapping of the O-antigen flippase (Wzx), polymerase (Wzy), and ligase (WaaL) from Pseudomonas aeruginosa $\mathrm{PAO} 1$ reveals novel domain architectures. mBio 1(3), e00189-10 (2010).

50 Daniels C, Vindurampulle Ch, Morota R. Overexpression and topology of the Shigella flexneri $\mathrm{O}$-antigen polymerase ( $\mathrm{Rfc} / \mathrm{Wzy})$. Mol. Microbiol. 28(6), 1211-1222 (1998).

51 Rush JS, Alaimo C, Robbiani R, Wacker M, Waechter Ch J. A novel Epimerase that converts GlcNAc-P-P-undecaprenol to GalNAc-P-P-undecaprenol in Escherichia coli O157*. J. Biol. Chem. 285(3), 1671-1680 (2010).
52 Amor PA, Yethon JA, Monteiro MA, Whitfield C. Assembly of the K40 antigen in Escherichia coli: identification of a novel enzyme responsible for addition of L-serine residues to the glycan backbone and its requirement for $\mathrm{K} 40$ polymerization. J. Bacteriol. 181(3), 772-780 (1999).

53 Stevenson G, Andrianopoulos K, Hobbs M, Reeves PR. Organization of the Escherichia coli $\mathrm{K}-12$ gene cluster responsible for production of the extracellular polysaccharide colanic acid. J. Bacteriol. 178(16), 4885-4893 (1996).

54 Morona R, Mavris M, Fallarino A, Manning $\mathrm{PA}$. Characterization of the $\mathrm{rfc}$ region of Shigella flexneri. J. Bacteriol. 176(3), 733-747 (1994).

55 Marolda CL, Vicarioli J, Valvano MA. Wzx proteins involved in biosynthesis of $\mathrm{O}$ antigen function in association with the first sugar of the O-specific lipopolysaccharide subunit. Microbiology 150 (Pt 12), 4095-4105 (2004).

56 Hong Y, Morcilla VA, Liu MA, Russell EL, Reeves PR. Three Wzy polymerases are specific for particular forms of an internal linkage in otherwise identical $\mathrm{O}$ units. Microbiology 161(8), 1639-1647 (2015). 